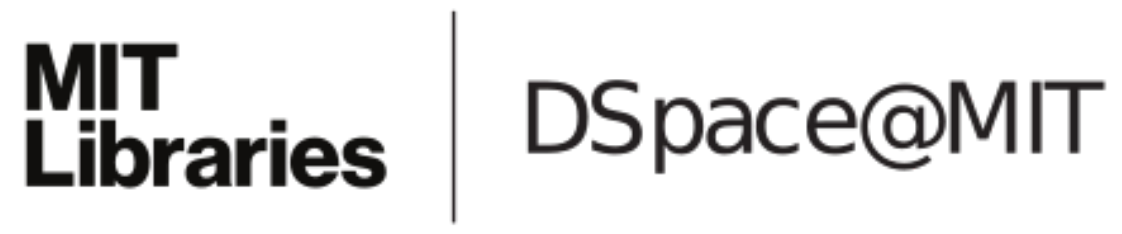

\author{
MIT Open Access Articles
}

A dynamic random access game with energy constraints

The MIT Faculty has made this article openly available. Please share how this access benefits you. Your story matters.

Citation: Altman, E. et al. "A dynamic random access game with energy constraints." Modeling and Optimization in Mobile, Ad Hoc, and Wireless Networks, 2009. WiOPT 2009. 7th International Symposium on. 2009. 1-7. ( 2009 IEEE

As Published: http://dx.doi.org/10.1109/WIOPT.2009.5291611

Publisher: Institute of Electrical and Electronics Engineers

Persistent URL: http://hdl.handle.net/1721.1/58811

Version: Final published version: final published article, as it appeared in a journal, conference proceedings, or other formally published context

Terms of Use: Article is made available in accordance with the publisher's policy and may be subject to US copyright law. Please refer to the publisher's site for terms of use. 


\title{
A Dynamic Random Access Game with Energy Constraints
}

\author{
Eitan Altman*,Tamer Başar ${ }^{\dagger}$, Ishai Menache ${ }^{\ddagger}$ and Hamidou Tembine* \\ * INRIA, MAESTRO Group, Sophia-Antipolis \\ $\dagger$ Department of Electrical and Computer Engineering, University of Illinois at Urbana-Champaign \\ $\ddagger$ Laboratory for Information and Decision Systems, Massachusetts Institute of Technology
}

\begin{abstract}
We study a dynamic random access game with a finite number of opportunities for transmission and with energy constraints. We provide sufficient conditions for feasible strategies and for existence of Nash-Pareto solutions and show that finding Nash-Pareto policies of the dynamic random access game is equivalent to partitioning the set of time slot opportunities with constraints into a set of terminals. We further derive upper bounds for pure Nash-Pareto policies, and extend the study to non-integer energy constraints and unknown termination time, where Time Division Multiplexing policies can be suboptimal. We show that the dynamic random access game has several strong equilibria (resilient to coalition of any size), and we compute them explicitly. We introduce the (strong) price of anarchy concept to measure the gap between the payoff under strong equilibria and the social optimum.
\end{abstract}

Index Terms-Dynamic game, multiple access control, strong equilibria, TDM policy.

\section{INTRODUCTION}

We examine how mobile terminals, aware of their remaining energy, adjust their individual transmission probabilities under orthogonal energy constraints (each mobile has energy constraints which depend only on its own control actions). The performance of each terminal in a multi-user system is significantly affected by the actions taken by other users. In recent years it has been advocated that for scalability reasons, centralized control of transmission power and/or other parameters should be replaced by autonomous distributed control to be performed by the mobiles in a decentralized fashion where each one may optimize its own performance. Much research has therefore been devoted over the last decade to the study of wireless networks using game-theoretic tools. In particular, several papers (e.g., [6], [3]) have studied the equilibrium properties of static random access games. The novelty of our approach here is in considering the dynamic game in which the probability of transmission of a terminal in slot $t$ depends on the amount of energy left prior to that slot.

\section{A. Contributions}

In the present paper we introduce a constrained dynamic game approach to study the equilibrium, optimality, and efficiency properties in random access scenarios. We consider a noncooperative game with $N$ terminals, where each terminal

This work has been done when the last co-author was visitor at University of Illinois at Urbana Champaign. This work was partially supported by an INRIA PhD internship grant. controls its energy and seeks to maximize its total payoff during the game. Our main contributions are the following: We provide explicit equilibrium characterization of the dynamic random access game with constraints. We show that the dynamic game has a finite number of constrained NashPareto equilibria and obtain some results on the number of such equilibria by using feasible Time Division Multiplexing (TDM) policies. We compute explicitly the (strong) price of anarchy, and determine the constrained strong equilibria. We show that TDM policies could be non-optimal in the noninteger energy constraints case and compute the equilibria for that case.

\section{B. Content}

The paper is structured as follows. The dynamic random access model is presented in Section II. Section III provides a comprehensive equilibrium analysis of the noncooperative game. We then examine in Section IV the efficiency loss in the network by using the concept of strong price of anarchy. In Section V, we consider the interesting case where the energy constraints are non-integers. Section VI concludes the paper.

\section{The Setting}

There are $N$ mobile terminals. Terminal $i$ has originally energy $\mathcal{E}^{i}$, and each transmission requires 1 unit of energy. Time is slotted. At time $t$ terminal $i$ transmits with some probability $p_{t}^{i}$. There are $m$ transmission opportunities (equivalently, the time horizon is $m$ ). A history $\mathcal{H}_{t}$ of length $t \leq m$ is a collection of vectors of probabilities of transmission from the initial slot to the time slot $t$. A behavioral strategy for player $i$ is a collection of $m$ maps labeled $t=1$ to $t=m$, where the $t$ th map is from the set $\mathcal{H}_{t}$ to a probability of a transmission at that time. A behavioral multi-strategy is a set of behavioral strategies, one for each player. Denote by $p^{i}=\left\{p_{t}^{i}\right\}_{t=1, \ldots, m}$ a behavioral strategy of terminal $i$. The vector of behavioral strategies of all $N$ terminals is denoted by $p=\left(p^{1}, \ldots, p^{N}\right)$. The total expected number of packets transferred successfully by terminal $i$ is given by

$$
\theta^{i}(p):=\sum_{t=1}^{m} p_{t}^{i} \prod_{j \neq i}\left(1-p_{t}^{j}\right)
$$


Let $Q^{i}$ be the total amount of energy used by terminal $i$ and let $\mathcal{Q}^{i}$ denote its expectation. Note that

$$
\mathcal{Q}^{i}(p)=\sum_{t=1}^{m} p_{t}^{i}
$$

The payoff of terminal $i$ is the expected number of packets successfully transmitted: $J^{i}(p)=h^{i}\left(\theta^{i}(p)\right)$, where $h^{i}: \mathbb{R} \longrightarrow \mathbb{R}$ is some increasing function. Terminal $i$ wishes to maximize this payoff subject to a constraint on the total (or total expected) energy consumption. This is formulated as:

$$
\text { P1(i): } \max _{\left\{p_{t}^{i}\right\}} J^{i}(p) \text { s.t } Q^{i} \leq \mathcal{E}^{i} P-a . s .
$$

An alternative problem is

$$
\text { P2(i): } \max _{\left\{p_{t}^{i}\right\}} J^{i}(p) \text { s.t } \mathcal{Q}^{i}(p) \leq \mathcal{E}^{i} .
$$

We let $P j:=\{P j(i), i=1,2, \ldots, N\}$.

\section{EQUILIBRIUM ANALYSIS}

In this section we analyze the equilibrium point of the underlying dynamic game. Specifically, in Sections III-A-III-B we focus on TDM policies, which are shown to be Paretoefficient. We consider other types of equilibria in Section III-C. We then introduce the notion of a strong equilibria and its relation to the social welfare problem. We conclude this section by considering the case when the termination time of the game is unknown in advance.

\section{A. Nash-Pareto Equilibria}

Recall that a policy $p$ is Nash equilibrium if it satisfies the energy constraint and if no terminal can benefit by deviating unilaterally. More precisely, if only player $i$ deviates then either its payoff does not increase, or its energy constraints are violated. A policy $p$ is Pareto efficient if it is feasible and there is no other policy $q$ that satisfies the energy constraints such that $J^{i}(q) \geq J^{i}(p)$ for all $i$ with strict inequality for at least one terminal $i$. A policy $p$ is a Nash-Pareto equilibrium if $p$ is a Nash equilibrium and $p$ is Pareto efficient. A Nash-Pareto policy may not exist in general, but we will show that such an equilibrium does exist in our case Note that if $m \geq \sum_{j=1}^{N} \mathcal{E}^{j}$ then it is possible to assign $N$ disjoint sets of slots to the $N$ players such that each player has sufficiently many slots to completely use his energy budget. Such a policy is obviously a Nash equilibrium and is Pareto efficient. We shall thus assume below that $m<\sum_{j=1}^{N} \mathcal{E}^{j}$. Throughout the bulk of this paper, we shall focus on the case where $\mathcal{E}^{j}$ are integers. Only in Section $\mathrm{V}$, non-integers $\mathcal{E}^{j}$ are briefly considered. We define below TDM policies, which play a major role in our equilibrium characterization.

Definition III-A1 (TDM policy). A TDM policy $p(M)$ is a partition of the set $\{1, \ldots, m\}$ into $N$ disjoint sets $M_{1}, \ldots, M_{N}$, such that at each time slot, $t \in\{1, \ldots, m\}$, the terminal ifor which $t \in M_{i}$ transmits a packet with probability 1 and all the others do not transmit. $p(M)$ is called feasible if $M_{i} \leq \mathcal{E}^{i}$ for each $i$.
Proposition III-A2. Any feasible TDM policy is a Nash equilibrium and is Pareto efficient for both problems PI and $P 2$.

Proof: Let $p$ be a feasible TDM policy. Since at each slot there is one transmission, any attempt of a player to deviate and transmit at a slot not assigned to it will result in a collision. Thus a player cannot increase its number of successful transmissions and hence its utility by attempting to use slots not assigned to it. It can gain neither by not transmitting at slots assigned to it. Hence $p$ is a Nash equilibrium. Assume next that $q=\left(q^{i}, p^{-i}\right)$ is another feasible TDM policy with $J^{i}(p)<J^{i}(q)$ for user $i$. This implies that for some other terminal $k, J^{k}(p)>J^{k}(q)$ since the total number of successful transmissions by all terminals is a constant $m$ under any feasible policy. This implies that $p$ is Pareto efficient.

Before proceeding with our game analysis, we provide below two simple numeric examples.

Example 1: $m=1, \mathcal{E} \geq 1$, The number of Nash equilibria is infinite (one of the terminals transmits with probability one and the others play any strategy). The number of Nash-Pareto solutions is exactly $N$. The game has a unique symmetric mixed equilibrium which consists of not transmitting with probability one (it is feasible from the assumptions that $\mathcal{E} \geq 1$ ). This implies that the symmetric equilibrium is not Pareto efficient.

Example 2: $m=4, N=2, \mathcal{E}^{1}=2, \mathcal{E}^{2}=3, \mathcal{E}^{1}+\mathcal{E}^{2}=$ $5>4=m$. There are $S(4,2)=7$ Nash-Pareto solutions without feasible permutations and $B_{4}=15: 4$ feasible TDM policies where the terminal one transmits only one time during all the four slots: $[\{1\},\{234\}],[\{2\},\{134\}],[\{3\},\{124\}]$, $[\{4\},\{123\}]$, and three TDM policies in which the terminal 1 transmits two times: $[\{12\},\{34\}],[\{13\},\{24\}],[\{14\},\{23\}]$. We should add three other feasible TDM policies obtained by feasible permutations between the two terminals: $[\{34\},\{12\}],[\{24\},\{13\}],[\{23\},\{14\}]$.

Based on combinatorial analysis, we are able to provide below a characterization of the number of TDM policies. The following lemma is immediate from combinatorial results on the partitions of a set (see [8], [5]).

Lemma III-A3. The number of TDM policies is exactly the Stirling number of the second kind $S(m, N)$ which is the number of partitions of a set of size $m$ into $N$ blocks $(N \leq m)$. $S(m, N)$ satisfies the recursive formula

$$
\begin{aligned}
S(m, N)= & \left(\begin{array}{l}
N \\
1
\end{array}\right) S(m-1, N)+S(m-1, N-1) \\
= & N S(m-1, N)+S(m-1, N-1), \\
& S(m, 1)=S(m, m)=1 .
\end{aligned}
$$

The Stirling numbers of the second kind are given by the explicit formula: $S(m, N)=\frac{1}{N !} \sum_{j=0}^{N}(-1)^{N-j} j^{m}\left(\begin{array}{c}N \\ j\end{array}\right)$.

It follows that the number of Nash-Pareto policies which guarantee at least one successful transmission is less than

$$
\frac{1}{N !} \sum_{j=0}^{N}(-1)^{N-j} j^{m}\left(\begin{array}{c}
N \\
j
\end{array}\right)
$$


without counting the feasible permutations between terminals in these policies.

Proposition III-A4. The total number of Nash-Pareto policies is less than the number of partitions of the set $\{1,2, \ldots, m\}$ to at most $N$ blocks. This number is given by $\sum_{j=1}^{N} S(m, j)$ which is lower than the $m$-th Bell number

$$
B_{m}=\sum_{j=0}^{m} S(m, j)
$$

for $m \leq N$. The Bell numbers satisfy the recursion formula:

$$
B_{m+1}=\sum_{j=0}^{m}\left(\begin{array}{c}
m \\
j
\end{array}\right) B_{j}
$$

Proof: Since the time horizon is finite, a Nash-Pareto policy must preserve a successful transmission at each time slot. Thus, a Nash-Pareto policy is necessarily a partition of $\{1,2, \ldots, m\}$ to $j$ non-empty blocks ( $j$ from one to $N$ ). The number of Nash-Pareto policies is exactly the number of

$$
\begin{gathered}
\left\{\left(M_{1}, \ldots, M_{k}\right) \mid \cup_{j=1}^{k} M_{j}=\{1, \ldots, m\},\right. \\
\left.1 \leq\left|M_{j}\right| \leq \mathcal{E}^{j}, M_{i} \cap M_{j}=\{\emptyset\} \text { if } i \neq j, k \leq N\right\}
\end{gathered}
$$

Hence, the number of partitions that are feasible is lower than $\sum_{j=1}^{N} S(m, j) \leq B_{m}$.

The total number of Nash-Pareto equilibria, considering also the order of the partitions (all the feasible permutations of the time slot of assignment of the terminal), is bounded by

$$
\sum_{j=1}^{N} j m ! S(m, j)
$$

\section{B. Symmetric energy constraints}

In this subsection, we assume that each terminal has originally the same energy $\mathcal{E}^{j}:=\mathcal{E} \geq 1, \forall j$. We obtain a recursion on the number of Nash-Pareto solutions of the dynamic random access game. Denote by $\operatorname{NP}(m, k, \mathcal{E})$ the number of Nash-Pareto that guarantees at least one successful transmission to each of the $k$ terminals $(m \geq k)$. Then the number of Nash-Pareto equilibria is exactly

$$
\mathrm{NP}(m, N)=\sum_{k=1}^{N} \mathrm{NP}(m, k, \mathcal{E}) .
$$

Proposition III-B1. The following recursive formula holds:

$$
\begin{gathered}
N P(m, j, \mathcal{E})=\left\{\begin{array}{cc}
0 & \text { if } \min (m, j) \leq 0 \\
0 & \text { if } m<k \text { or } m>\mathcal{E} k, \\
S(m, j) & \text { if } \mathcal{E}>m
\end{array}\right. \\
N P(m, k, \mathcal{E})=\sum_{j=0}^{-1+\mathcal{E}}\left(\begin{array}{c}
m-1 \\
j
\end{array}\right) N P(m-1-j, k-1, \mathcal{E})
\end{gathered}
$$

Proof: The first expression is immediate from the definitions. We now prove the second formula $\operatorname{NP}(m, k, \mathcal{E})=$ $\sum_{j=0}^{-1+\mathcal{E}}\left(\begin{array}{c}m-1 \\ j\end{array}\right) \mathrm{NP}(m-1-j, k-1, \mathcal{E})$. The left-hand side term counts exactly the number of partitions of the set $\{1,2, \ldots, m\}$ with the restriction that each block of the partition must have at most $\mathcal{E}$ elements. Select now the one element of $\{1,2, \ldots, m\}$ (says " 1 ") as a reference. Then the reference element "1" can have at most $\mathcal{E}$ other elements in the same blocks of partition. We then can can add $0,1, \ldots,-1+\mathcal{E}$ other elements in the partition. We count the number of partition when $j \in\{0,1,2, \ldots,-1+\mathcal{E}\}$ elements are added to " 1 ". This gives $\mathrm{NP}(m-1-j, k-1, \mathcal{E}) \times$ the number of choices of those $j$ elements between $m-1$, i.e. the binomial $\left(\begin{array}{c}m-1 \\ j\end{array}\right)$. The number $\operatorname{NP}(m-1-j, k-1, \mathcal{E})$ counts the number of partitions of the remaining elements $m-1-j$ into $k-1$ blocks with each block at most $\mathcal{E}$ elements. In total, one has

$$
\sum_{j=0}^{-1+\mathcal{E}}\left(\begin{array}{c}
m-1 \\
j
\end{array}\right) \mathrm{NP}(m-1-j, k-1, \mathcal{E}) .
$$

We now give a formula on the difference between the number of feasible TDM policies (FTDM) without exchanging the roles between terminals and the number of TDM policies in case of symmetric constraints:

$$
\begin{aligned}
|\operatorname{FTDM}(m, N, \mathcal{E})|= & |\operatorname{FTDM}(m-1, N-1, \mathcal{E})| \\
& +\left(\begin{array}{c}
N \\
1
\end{array}\right)|\operatorname{FTDM}(m-1, N, \mathcal{E})| \\
& -\left(\begin{array}{c}
m-1 \\
\mathcal{E}
\end{array}\right)|\operatorname{FTDM}(m-\mathcal{E}, N-1, \mathcal{E})|
\end{aligned}
$$

We can easily verify this formula for $m \leq 3$ or $\mathcal{E} \geq m$ (by Stirling number of second kind).

Example 3: $m=4, N=2, \mathcal{E}^{1}=2=\mathcal{E}^{2}$ (symmetric constraints). From the recursive formula with symmetric constraints, one has $\mathrm{NP}(4,2,2)=\mathrm{NP}(3,1,2)+$ $\left(\begin{array}{l}3 \\ 1\end{array}\right) \mathrm{NP}(2,1,2)$. The first term $\mathrm{NP}(3,1,2)=0$ because of the constraint at most 2 elements in the block. The term $\operatorname{NP}(2,1,2)=1$. We conclude that $\operatorname{NP}(4,2,2)=3$ which are the 3 TDM policies $([\{12\},\{34\}],[\{13\},\{24\}],[\{14\},\{23\}])$ and 3 others are obtained by exchanging the role of terminal 1 and terminal 2: $[\{34\},\{12\}],[\{24\},\{13\}],[\{23\},\{14\}]$. Similarly, $\mid$ FeasibleTDM $(3,1,2) \mid=0$, and $|\operatorname{FTDM}(3,1,2)|=$ 3 obtained from $[\{12\},\{3\}],[\{13\},\{2\}],[\{23\},\{1\}]$ and $|\operatorname{FTDM}(2,1,2)|=1$.

$$
\begin{aligned}
|\operatorname{FTDM}(4,2,2)|= & |\operatorname{FTDM}(3,1,2)| \\
& +\left(\begin{array}{l}
2 \\
1
\end{array}\right)|\operatorname{FTDM}(3,2,2)| \\
& -\left(\begin{array}{l}
3 \\
2
\end{array}\right)|\operatorname{FTDM}(2,1,2)|
\end{aligned}
$$

i.e., $3=0+2 \times 3-\left(\begin{array}{l}3 \\ 2\end{array}\right)=6-3=3$.

\section{Non-TDM Equilibria}

Proposition III-C1. The constrained dynamic random access game has an infinite number of equilibria in behavioral strategies.

Proof: Since $m<\sum_{j=1}^{N} \mathcal{E}^{j}$, there exists a feasible strategy at which at each time slot, at least one of the $N$ users 
transmits with probability one. Then, any other feasible strategy profile of the other terminals leads to an equilibrium. Since the strategies in the form $\left(p_{t}^{1}, \ldots, p_{t}^{j-1}, 1, p_{t}^{j+1}, \ldots, p_{t}^{N}\right)$ are equilibria of the one-shot game at time slot $t$, it follows that there is an infinite number of equilibria in behavioral mixed strategies.

To further describe equilibria that are not of TDM nature, we define the following types of equilibria: An equilibrium is completely mixed (or fully mixed) if $0<p_{t}^{i}<1$ for all $i, t$. An equilibrium is partially mixed if at least one player uses pure strategy, at least one player has completely mixed strategy, the others players has arbitrary strategies. We have the following result.

Proposition III-C2. The game has no interior equilibria in asymmetric behavioral strategies which are Pareto optimal. Moreover, if the strategy profile $p^{-j}=$ $\left(p^{1}, \ldots, p^{j-1}, p^{j+1}, \ldots, p^{N}\right)$ is a completely mixed behavioral strategy profile of the all terminals other than $j$, i.e., $\forall k, t, 0<p_{t}^{k}<1, \sum_{t=1}^{m} p_{t}^{k} \leq \mathcal{E}^{k}$, then the best strategy that the terminal $j$ can use is given by

$$
\left\{\begin{array}{c}
\text { transmit w.p } 1 \text { if } \\
\text { not transmit }
\end{array} \quad \begin{array}{c}
t \in \mathcal{B}\left(\min \left(\mathcal{E}^{j}, m\right)\right), \\
\text { otherwise }
\end{array}\right.
$$

where $\mathcal{B}(k)$ is the set of time slots that determine the $k$ first highest elements in the set $\left\{\prod_{i \neq j}\left(1-p_{t}^{i}\right)\right\}_{1 \leq t \leq m}$.

Proof: Let $C_{t}^{-j}=\prod_{i \neq j}\left(1-p_{t}^{i}\right)$. Since $\forall i \neq j, 0<p_{t}^{i}<$ 1 , one has $0<C_{t}^{-j}<1$ for every time slot. There exists a permutation $\varphi$ that orders the elements of $\left\{C_{t}^{-j}\right\}_{t}$ as

$$
C_{\varphi(1)}^{-j} \geq C_{\varphi(2)}^{-j} \geq \ldots \geq C_{\varphi(m)}^{-j} .
$$

Since the maximum of time slots (delay) is $m$, the terminal $j$ can invest at most $\min \left(\mathcal{E}^{j}, m\right)$. Since $\mathcal{E}^{j} \in \mathbb{Z}_{+}$, the set of time slots that gives in order the $\min \left(\mathcal{E}^{j}, m\right)$ highest elements in $\left\{C_{t}^{-j}\right\}$ is given by $\mathcal{B}\left(\min \left(\mathcal{E}^{j}, m\right)\right)=\left\{t \mid C_{t}^{-j} \geq\right.$ $\left.C_{\varphi\left(\min \left(\mathcal{E}^{j}, m\right)\right)}^{-j}\right\}=\left\{\varphi(1), \ldots, \varphi\left(\min \left(\mathcal{E}^{j}, m\right)\right)\right\}$ and the best response to $p^{-j}$ is to transmit at any time $t \in \mathcal{B}\left(\min \left(\mathcal{E}^{j}, m\right)\right)$ and stay quiet otherwise. The energy constraint is satisfied and the payoff is maximized at that strategy. The maximum payoff of $j$ is $h^{j}\left(\sum_{t \in \mathcal{B}\left(\min \left(\mathcal{E}^{j}, m\right)\right)} C_{t}^{-j}\right)$. Thus the terminal $j$ is not indifferent between transmit and not transmit when the others terminals use $p^{-j}$ because he gets more when he transmits at the time slots in $\mathcal{B}\left(\min \left(\mathcal{E}^{j}, m\right)\right)$. Hence there is no interior strategy which is best response to $p^{-j}$ for any $j$. We conclude that the game has no interior equilibrium in asymmetric behavioral strategies.

Corollary III-C3. The same argument as in Proposition III-C2 holds if the strategy profile $p$ satisfies $0 \leq p_{t}^{i}<1, \forall i, t$.

At such an equilibrium, at least one of the terminals transmits with probability one at any time slot. If $p_{t}^{i}=1$ for some $t$, then $C_{t}^{-j}=0$ and any strategy of terminal $j$ at time $t$ yields a payoff of zero. Hence $p_{t}$ has the form, $\left(q_{t}^{1}, q_{t}^{2}, \ldots, q_{t}^{N}\right)$ with $\prod_{k=1}^{N}\left(1-q_{t}^{k}\right)=0$. The partially mixed equilibria are not Pareto optimal as shown in Theorem III-C5.

Proposition III-C4. (Symmetric time independent interior equilibrium) Suppose that $\mathcal{E}^{j}=\mathcal{E}$ for all $j$.
- If $\mathcal{E}^{j}=\mathcal{E}<m<N \mathcal{E}$, then the interior strategy profile $p$ defined by $p_{t}=\left(\frac{\mathcal{E}}{m}, \ldots, \frac{\mathcal{E}}{m}\right)$ for each $t$ is a Nash equilibrium.

- If $\mathcal{E}>m$, then there is no symmetric interior equilibrium

Proof: The energy constraints are satisfied $0 \leq \frac{\mathcal{E}}{m} \leq$ $1, \mathcal{Q}^{j}(p)=\frac{\mathcal{E}}{m} \times m=\mathcal{E}, \forall j$ and it is not difficult to see that the strategy $p^{j}=\left(\frac{\mathcal{E}}{m}, \ldots, \frac{\mathcal{E}}{m}\right)$ is a best reply to itself. Suppose that $\mathcal{E}>m$. Then, any symmetric time independent interior strategy $x$ uses less energy and the expected number of successful transmissions is $m x(1-x)^{N-1}$ which is strictly lower than $m(1-x)^{N-1}$ which is the payoff obtained when the terminal transmits at each time slot. Hence, $x$ is not a best response to itself.

Proposition III-C5. The (partially) mixed equilibria of the game are not Pareto efficient.

Proof: By Proposition III-C2 and Corollary III-C3, if more than two terminals transmit with probability one at the same time slot then the policy does not lead to a Pareto optimal one. It suffices to prove the result for the case where only one terminal $i$ transmits, another one $j$ uses $0<p_{t}^{j}<1$ and the others use a probability strictly lower than one. The strategy $p$ is then Pareto dominated by $q$ where $q_{t}$ is $q_{t}=\left(p_{t}^{1}, \ldots, p_{t}^{i-1}, 1, p_{t}^{i+1}, \ldots, p_{t}^{j-1}, 0, p_{t}^{j+1}, \ldots, p_{t}^{N}\right):$

(i) Under the strategy $q$ the payoff of all terminals other than $i$ remain the same. (ii) The payoff of $j$ is unchanged at time slot $t$ because there is collision if $j$ transmits at time $t$. (iii) The total number of successful transmissions of $i$ will increase by $\prod_{k \neq i, j}\left(1-p_{t}^{k}\right) \times(1-0)-\prod_{k \neq i, j}\left(1-p_{t}^{k}\right) \times\left(1-p_{t}^{j}\right)=$ $p_{t}^{j} \prod_{k \neq i, j}\left(1-p_{t}^{k}\right)>0$. Since $h^{i}$ is strictly increasing, $q$ is better than $p$ in the Pareto sense.

\section{Strong equilibria}

A policy is a $k$-strong (Nash) equilibrium if no coalition of size $k$ can improve the number of successful transmissions of each of its members. A policy is a strong equilibrium if it is a $k$-strong equilibrium for any size $k$. A strong equilibrium is then a policy from which no coalition (of any size) can deviate and improve the payoff of every member of the coalition, while possibly lowering the payoff of terminals outside the coalition group. This notion of strong equilibria is very attractive because it is resilient against coalitions of terminals. Most of the games do not admit any strong equilibrium but in our case we will show that the dynamic random access game has many strong equilibria.

Proposition III-D1. The feasible TDM policies that we extend to empty set of the partition (or the number of blocks is at most $N)$ are strong equilibria.

Proof: Let $p$ be a feasible extended TDM policy (i.e., where $\left|M_{j}\right| \geq 0$ ). Since at each slot there is exactly one transmission, any attempt of a coalition of any size $k \leq N$ of terminals to deviate and transmit at a slot not assigned to it will result in a collision. Thus the coalition of players cannot increase its number of successful transmission and hence the payoffs of its members by attempting to use slots not assigned to it. It can neither gain by not transmitting at slots assigned 
to it. Since the total number of successful transmissions by all terminals is $m$ under the feasible policy $p$ which is the maximum number of successful transmissions that we have during the game, the policy $p$ is a then strong equilibrium.

To be a strong equilibrium, a strategy profile must necessarily satisfy the property that there is no collision at any time slot. This implies that at each time slot, only one terminal transmits and the others stay quiet (under the energy constraints). We conclude that strong equilibria are extended TDM policies. Note that in general a Nash-Pareto equilibrium may not be a strong Nash equilibrium, but in our case, we then have the following result:

Proposition III-D2. In the dynamic game, the extended TDM policies, the strong equilibria and the Nash-Pareto equilibria coincide.

\section{E. Maximization of successful transmissions}

Any pure Pareto-Nash equilibrium of the dynamic game corresponds to, the same number of successful transmissions $m$ which is the maximum number of successful transmissions in $m$ time slots. Thus, the feasible TDM policies and other Pareto-Nash policies (the time divisions can be least than $N$ groups) described above solve the maximization problem:

$$
\begin{gathered}
\max _{p=\left(p^{1}, \ldots, p^{N}\right)} \mathcal{V}(p):=\sum_{j=1}^{N} J^{j}(p) \text { subject to } \\
\left\{\begin{array}{c}
Q^{j}(p) \leq \mathcal{E}^{j} \\
j=1, \ldots, N .
\end{array}\right.
\end{gathered}
$$

which gives a social welfare (or global optimum). When the $h^{j}$ are the identity functions, then the optimality and the efficiency of strong equilibria of the dynamic game can be characterized as follows: Any strong equilibrium of the dynamic game is a social welfare. In addition, a strong equilibrium is also an energy efficient equilibrium in the sense that the equilibrium is realized with a minimum energy investment for each user and it is a social welfare. Figure 1 (resp. 2) represents the total expected number of successful transmissions respectively for 2,10,50 time slots when $N=10$ (resp. for $N=4,20,50$ terminals when $m=50$ ) in symmetric time independent strategies. We can see from these figures that if the strategy $\frac{1}{N}$ is feasible then it is a social welfare in symmetric time independent strategies.

\section{F. Unknown Termination Time}

We conclude this section by briefly considering the case where the game terminates at some time $T$, which the terminals do not know in advance. That is, we assume that $T$ is a random variable, independent of the actions of terminals, taking values in $\{1,2 \ldots,-1+$ $\left.\sum_{j=1}^{N} \mathcal{E}^{j}\right\}$. The expected payoff of terminal $j$ is $J^{j}(p)=$ $h^{j}\left(\mathbb{E}\left(\theta^{j, T}\right)\right)=h^{j}\left(\sum_{m \geq 1} P(T=m) \theta^{j, m}(p)\right)$ where $\theta^{j, m}(p)=\sum_{t=1}^{m} p_{t}^{j} \prod_{k \neq j}\left(1-p_{t}^{k}\right)$ Note that if $T$ obeys a geometric distribution with parameter $0 \leq \alpha<1$ (without energy constraints) $P(T=m)=(1-\alpha) \alpha^{m-1}, m \geq 1$ we obtain the

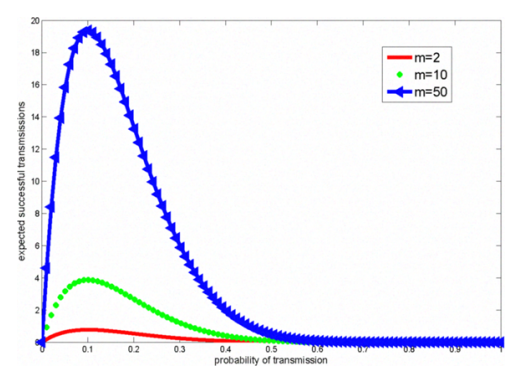

Fig. 1. Global optimum : $m=2,10,50, N=10$.

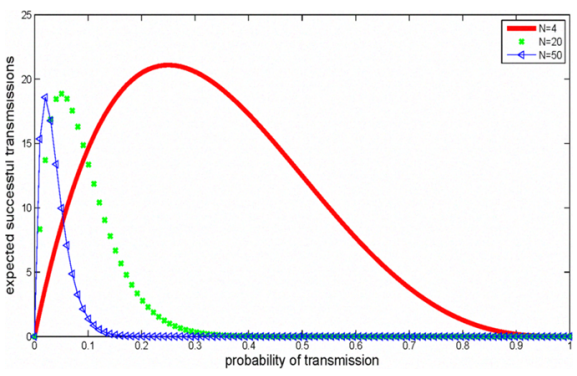

Fig. 2. Global optimum: $N=4,20,50$.

so-called discounted payoff. That is, the communication will be terminated with probability $(1-\alpha)$ at each time slot. If $h^{j}$ is convex, then $J^{j}(p) \leq \sum_{m>1} P(T=m) h^{j}\left(\theta^{j, m}(p)\right)$. It is clear that any feasible strategy that assigns each time to a terminal with probability one is still an equilibrium. This strategy profile depends only on $\sum_{j=1}^{N} \mathcal{E}^{j}$, but is independent of the random variable $T$. Thus, the expected number of successful transmissions under this strategy is $\mathbb{E}(T)=\sum_{m \geq 1} m P(T=m)$ (the game can terminate before that some assigned terminals transmit). We shall not pursue further the unknown termination time case, and leave the full analysis thereof to future work.

\section{Strong Price of Anarchy}

Throughout this section, we assume that the $h^{j}$ are the identity functions. One of the metrics used to measure how much the performance of decentralized systems is affected by the selfish behavior of its components is the so-called price of anarchy. We present here the analogue of price of anarchy for the maximization problem using strong equilibria and TDM policies. The Price of Anarchy can be seen as an efficiency metric that measures the price of selfishness or decentralization and has been extensively used in the context of congestion games or routing games where typically users haves to minimize a cost function. In the context of random access, we define an equivalent measure of price of anarchy for maximization problems. To have an interpretation as a price, the inverse of the ratio that we define or the difference between 1 and the ratio (normalized gap measure), are more appropriate. Then, the infinite price of anarchy " $\frac{1}{0}$ " can be seen as the largest price of anarchy. Also, the indetermination 
disappears by translation of the payoff function (it is known that this operation does not change the set of equilibria).

One of the advantages of a strong equilibrium is that it has a potential to reduce the distance between the optimal solution and the solution obtained as an outcome of selfish behavior, typically in the case where there is a collision at each time slot. Since the dynamic game has strong equilibria, we can define the strong price of anarchy, introduced in [1], as the ratio between the payoff of the worst strong equilibrium and the social optimum value.

Proposition IV-A. For the dynamic random access game with the number of successful transmissions as the payoff, the strong price of anarchy is one.

Proof: Since the strong equilibria are extended TDM policies, they lead to the same number of successful transmissions, $m$. Strong Price of Anarchy $=\min _{p \in \operatorname{SE}} \frac{\sum_{j=1}^{N} J^{j}(p)}{S W}=1$ where $\mathrm{SE}$ is the set of strong equilibria, $S W$ is the payoff obtained at any feasible social welfare value: $S W:=$ $\max _{q} \mathcal{V}(q)$ s.t $\sum_{t=1}^{m} q_{t}^{j} \leq \mathcal{E}^{j}, \forall j \in\{1, \ldots, N\}$. Hence, ratio between the payoff of the worst strong equilibrium and the feasible social welfare value is one.

Note that when $2 m<\sum_{j=1}^{N} \mathcal{E}^{j}$, there exists a feasible strategy profile for which at least two terminals can transmit in the same time slot, for any time slot. The strategy profile where there is collision in each time slot leads to a Nash equilibrium. Then the worst Nash outcome is zero and the price of anarchy too: Price of Anarchy $=\min _{p \in N E} \frac{\sum_{j=1}^{N} J^{3}(p)}{S W}=0$. This indicates that the efficiency of decentralization is $0 \%$ i.e., complete inefficiency. The optimistic price of anarchy of the best constrained Nash equilibrium also called price of stability [2] is also 1, i.e. the efficiency of TDM policies is $100 \%$. Price of Stability $=\max _{p \in N E} \frac{\sum_{j=1}^{N} J^{j}(p)}{S W}=1=$ Strong Price of Anarchy.

We have seen that the price of anarchy captures the efficiency of all the system, but what is the individual efficiency of each terminal in that contribution? Clearly, the maximum payoff that terminal $i$ can gain under the energy constraint is $h^{i}\left(\min \left(\mathcal{E}^{i}, m\right)\right)$. But the payoff that terminal $i$ can obtain in Nash-Pareto equilibria or correlated equilibria can be less that $h^{i}\left(\min \left(\mathcal{E}^{i}, m\right)\right)$. This motivates us to define the individual efficiency metric (IEM) for strong equilibria. The individual efficiency metric captures the price of non-cooperation (in terms of energy investment or in terms of payoff) for each terminal $i$. The strong IEM of terminal $i$ is then the ratio between the worst payoff of $i$ and the maximum payoff that $i$ can gain, i.e. $\operatorname{IEM}^{i}=\min _{p \in \text { TDM policies }} \frac{J^{i}(p)}{h^{i}\left(\min \left(\mathcal{E}^{i}, m\right)\right)}$. It is easy to see that the strong $\operatorname{IEM}^{i}$ is zero. This says that the efficiency of $i$ can also be $0 \%$, even when the strong price of anarchy is 1 .

\section{NON-INTEGER ENERGy CONSTRAINTS}

The TDM policies are based on total assignment of the $m$ time slots to the terminals. Suppose now that the $\mathcal{E}^{j}$ are not necessarily integers. Denote by $\lfloor x\rfloor$ the integer part of $x$. We observe that FeasibleTDM $\left(m, N, \mathcal{E}^{1}, \mathcal{E}^{2}, \ldots, \mathcal{E}^{N}\right)=$
FeasibleTDM $\left(m, N,\left\lfloor\mathcal{E}^{1}\right\rfloor,\left\lfloor\mathcal{E}^{2}\right\rfloor, \ldots,\left\lfloor\mathcal{E}^{N}\right\rfloor\right)$ which follows from the complete assignment (with probability one) of the time slot to a terminal when it is feasible. We address now the following question: Are feasible TDM policies optimal if the vector of constraints are not natural integers? $\left(\mathcal{E}^{1}, \mathcal{E}^{2}, \ldots, \mathcal{E}^{N}\right) \notin \mathbb{Z}_{+}^{N}$ i.e., do we obtain the same optimal value by solving with the constraints:

$$
\left\{\begin{array}{c}
Q^{j}(p) \leq\left\lfloor\mathcal{E}^{j}\right\rfloor \\
1 \leq j \leq N
\end{array}\right.
$$

We show that the answer is negative. Feasible TDM policies can be suboptimal and may not be Nash equilibria in the noninteger case. The following example illustrates this assertion. Example 4: TDM policies are not Nash equilibria and are not efficient. Consider the following parameters: $m=4, N=$ $4, \mathcal{E}^{1}=2, \mathcal{E}^{j}=0.9999+\frac{10^{-6}}{j}, j>1$. We verify that $\sum_{j} \mathcal{E}^{j}=2+3(0.9999)+10^{-6}\left(\frac{1}{2}+\frac{1}{3}+\frac{1}{4}\right)=4.9997+$ $\frac{13}{12} 10^{-6}>4=m$. Since $\left\lfloor\mathcal{E}^{j}\right\rfloor=0$, for all $j \geq 2$, and $\mathcal{E}^{1}=2$, the extended feasible TDM policies assign two of the four time slots to terminal 1 and there is no transmission for the rest of the time slots. There are 6 possibilities to do such assignments. The total payoff obtained by any feasible TDM policy is then $h^{1}(2)$. The following proposition shows that it is possible to gain more than $h^{1}(2)$ as a total payoff.

Proposition V-A. The following holds: (a) feasible TDM policies are not Pareto optimal, (b) feasible TDM policies are not equilibria.

Proof: Let $p$ be a feasible TDM policy that assigns to terminal 1 the two first slots. Consider the following policy $q^{*}$ : (a) $q^{*}$ assigns the two first slots to terminal 1 , (b) at slot three, terminal 2 transmits with probability $\mathcal{E}^{2}$, (c) at slot four, terminal 3 transmits with probability $\mathcal{E}^{3}$. We show that $q^{*}$ Pareto dominates $p$ and $p$ is not a Nash equilibrium. The payoff obtained at $q^{*}$ is $h^{1}(2)$ for terminal $1, h^{2}\left(\mathcal{E}^{2}\right)>0=h^{2}(p)$ for terminal 2, and $h^{3}\left(\mathcal{E}^{3}\right)>0=h^{3}(p)$ for terminal 3 , and terminal 4 gets zero. We conclude $q^{*}$ Pareto dominates $p$. Moreover the best response to $p^{-j}$ for a terminal $j \geq 2$ is to transmit with some probability $0<q^{j}<\mathcal{E}^{j}$ at least at one of the two slots where the terminal 1 is not assigned to. Thus, $p$ is not an equilibrium. The same argument works for the others 5 policies.

Proposition V-B. A necessary and sufficient condition for the existence and optimality of feasible TDM policies in the dynamic random access game is $\sum_{j=1}^{N}\left\lfloor\mathcal{E}^{j}\right\rfloor \geq m$.

Proof: Necessity: The necessity to have this condition comes from the Example 3. If this condition is not satisfied then by Example 3 we get a counterexample where feasible TDM policies are not efficient and not in equilibrium.

Sufficiency: If $\sum_{j=1}^{N}\left\lfloor\mathcal{E}^{j}\right\rfloor \geq m$, then the existence of feasible TDM policies that cover all the time slots is guaranteed. The optimality and equilibria follow by Theorems III-A2, III-D2 and the arguments in Subsection III-E. This completes the proof.

In the remainder of this section, we consider Nash equilibria when $\sum_{j=1}^{N}\left\lfloor\mathcal{E}^{j}\right\rfloor<m$ and $\sum_{j=1}^{N} \mathcal{E}^{j} \geq m$. Let $m^{\prime}:=m-k$ where $k$ is the maximum number of slots that can be assigned, 
i.e. $k=\sum_{j=1}^{N}\left\lfloor\mathcal{E}^{j}\right\rfloor$. We have seen that if $k \geq m$ there exists a feasible TDM policy which is a strong equilibrium and it is a social welfare. Suppose now that $\sum_{j=1}^{N}\left\lfloor\mathcal{E}^{j}\right\rfloor<m$ and $\sum_{j=1}^{N}\left\lfloor\mathcal{E}^{j}\right\rfloor \geq m$. Consider the following strategy:

- The $k$ first time slots are assigned by a feasible policy $p$.

- Let $\mathcal{N}^{\prime}$ be the set of terminals $j$ for which $\mathcal{E}^{j} \notin \mathbb{Z}_{+}$. Let $N^{\prime}=$ cardinal of $\mathcal{N}^{\prime}$. The number $N^{\prime} \geq 1$ by assumption. For all $j \in \mathcal{N}^{\prime}, \overline{\mathcal{E}}^{j}:=\mathcal{E}^{j}-\left\lfloor\mathcal{E}^{j}\right\rfloor$ is positive and strictly lower than one. The terminals which are not in $\mathcal{N}^{\prime}$ has zero energy at the end the slot $k$.

- From $k+1$ to $m$, each terminal $j \in \mathcal{N}^{\prime}$ has its total remaining energy $\overline{\mathcal{E}}^{j} \in(0,1)$. Each terminal solves the following constrained optimization problem for the remaining slots:

$$
\begin{gathered}
\operatorname{SubProblem}(\mathrm{k}, \mathrm{j}), j \in \mathcal{N}^{\prime}: \\
\max _{\left\{p_{t^{\prime}}^{j}\right\}_{t^{\prime} \geq k+1}} h^{j}\left(\sum_{t=k+1}^{m} p_{t}^{j} \prod_{i \in \mathcal{N}^{\prime} \backslash\{j\}}\left(1-p_{t}^{i}\right)\right) \text { s.t } \\
0 \leq \sum_{t=1} p_{t}^{j} \leq \mathcal{E}^{j}-\left\lfloor\mathcal{E}^{j}\right\rfloor<1 .
\end{gathered}
$$

Proposition V-C. Let $s=\left(p_{1}, \ldots, p_{k}, q_{k+1}, \ldots, q_{m}\right)$ with

$$
\left\{\begin{array}{cc}
q_{t+k}^{j}=\frac{\overline{\mathcal{E}}^{j}}{m^{\prime}}=\frac{\mathcal{E}^{j}-\left\lfloor\mathcal{E}^{j}\right\rfloor}{m-\sum_{j=1}^{N}\left\lfloor\mathcal{E}^{j}\right\rfloor} & \text { if } j \in \mathcal{N}^{\prime} \\
0 & j \notin \mathcal{N}^{\prime} .
\end{array}\right.
$$

Then,

- The strategy s is a Nash equilibrium of the dynamic game.

- $s$ is not a global optimum.

Proof: We show that $s$ is a Nash equilibrium. For $t \leq k$, it is clear that unilateral deviation is non-profitable. For $t \geq k+1$, define Lagrange function for each terminal SubProblem $(\mathrm{k}, \mathrm{j}), \quad j \in \mathcal{N}^{\prime}: L^{j}\left(\left\{q_{t}^{j}\right\}_{t \geq k+1}, \lambda^{j}\right)=$ $h^{j}\left(\sum_{t=k+1}^{m} q_{t}^{j} \prod_{i \in \mathcal{N}^{\prime} \backslash\{j\}}\left(1-q_{t}^{i}\right)\right)-\lambda^{j}\left(\sum_{t=1}^{m} q_{t}^{j}-\overline{\mathcal{E}}^{j}\right)$. The Karush-Kuhn-Tucker first optimality conditions give $\forall j \in \mathcal{N}^{\prime}$, $h^{\prime j}\left(\sum_{t=k+1}^{m} q_{t}^{j} \prod_{i \in \mathcal{N}^{\prime} \backslash\{j\}}\left(1-q_{t}^{i}\right)\right) \prod_{i \in \mathcal{N}^{\prime} \backslash\{j\}}\left(1-q_{t}^{i}\right)=$ $\lambda^{j}, \sum_{j=k+1}^{m} q_{t}^{j}=\mathcal{E}^{j}-\left\lfloor\mathcal{E}^{j}\right\rfloor, q_{t}^{j} \geq 0$. After some computations, we derive that the strategy in which each terminal $j \in \mathcal{N}^{\prime}$ uses its remaining energy $\mathcal{E}^{j}-\left\lfloor\mathcal{E}^{j}\right\rfloor$ uniformly in the $\mathrm{m}^{\prime}$ slots, solves the above problem and the fixed-point equation of the best-response correspondence. Let $T_{1}:=\left\{j, \overline{\mathcal{E}}^{j}=\right.$ $\left.\max _{i} \overline{\mathcal{E}}^{i}\right\}$, be the set of terminals with the maximum energy available after the $k$ slots and $\tau_{1}$ the cardinal of $T_{k+1}$. If $\tau_{1}<m^{\prime}$, define $T_{2}:=\left\{j \notin T_{1}, \overline{\mathcal{E}}^{j}=\max _{i \notin T_{1}} \overline{\mathcal{E}}^{i}\right\}$, the second best maxima of energy after the $k$ slots. More generally, we define

$$
T_{l}:=\left\{j \notin \cup_{l^{\prime}=1}^{l-1} T_{l^{\prime}}, \overline{\mathcal{E}}^{j}=\max _{i \notin \cup_{l^{\prime}}^{l-1} T_{l^{\prime}}} \overline{\mathcal{E}}^{i}\right\}
$$

the $l$-best maxima of $\mathcal{E}^{j}-\left\lfloor\mathcal{E}^{j}\right\rfloor$. Consider a policy $s^{\prime}$ that uses $s_{t}^{\prime}=p_{t}$ in the $k$ first slots and from $k+1$ to $k+\tau_{1} \leq$ $m$ one of the terminals $j \in \mathcal{N}^{\prime}$, transmits with probability $s^{\prime j}{ }_{t^{\prime}+k}^{j}=\max _{i \in T_{1}}\left(\mathcal{E}^{i}-\left\lfloor\mathcal{E}^{i}\right\rfloor\right)$ and others do not transmit. From $k+\tau_{1}+1$ to $k+\tau_{2} \leq m$, one of the terminals $j \in \mathcal{N}^{\prime}$ transmits with probability $s_{t^{\prime}+k}^{\prime j}=\max _{i \in T_{2}}\left(\mathcal{E}^{i}-\left\lfloor\mathcal{E}^{i}\right\rfloor\right)$, and others do not transmit, and so on. The two strategies $s^{\prime}$ and $s$ coincide at the first $k$ slots. The social payoff obtained between the slots $k$ and $m$ under $s^{\prime}$ is strictly greater than the social payoff obtained under $s$. Hence, $s$ is not a global optimum.

\section{CONCLUDING REMARKS}

In this paper we have studied a dynamic random access game with energy constraints. We have shown that the game has an infinite number of Nash equilibria and an exponential number of strong equilibria which we compute explicitly. In the integer case we showed that any feasible strategy profile that assigns a time slot to a single terminal leads to a Nash equilibrium which is Pareto optimal. In the non-integer case, we demonstrated that TDM can be suboptimal and infeasible. Finally, we have analyzed the efficiency of these equilibria using price of anarchy and a specific individual efficiency metric. An interesting future direction would be to consider multi-packet reception models, where the transmission success probability decreases with the number of simultaneous transmissions.

\section{REFERENCES}

[1] N. Andelman, M. Feldman, and Y. Mansour, Strong Price of Anarchy. In SODA'07, 2007.

[2] E. Anshelevich, A. Dasgupta, J. Kleinberg, E. Tardos, T. Wexler and T. Roughgarden, The Price of Stability for Network Design with Fair Cost Allocation. In 45th Annual IEEE Symposium on Foundations of Computer Science (FOCS), pages 59-73, 2004.

[3] L. Chen, S. H. Low and J. C. Doyle, Random Access Game and Medium Access Control Design, 2006.

[4] A.J. Hoffman, J.B. Kruskal, Integral Boundary Points of Convex Polyhedra, in Kuhn H.W. ,Tucker, A.W., Linear Inequalities and Related Systems, Annals of Mathematics Studies, 38, Princeton: Princeton University Press, pp. 223-246, 1956.

[5] P. Hilton, J. Pedersen, J. Stigter, On partitions, surjections, and Stirling numbers, Bull. Belg. Math. Soc. 1 ,713-725, 1994.

[6] H. Inaltekin and S.B. Wicker, A One-shot Random Access Game for Wireless Networks- Behavior of Nodes at Nash Equilibria," IEEE/ACM Trans. on Networking, December 2008.

[7] A. W. Marshall and I. Olkin , Inequalities: Theory of of Majorization and Its Applications, Academic Press, 1979.

[8] G.-C. Rota , 1964, The Number of Partitions of a Set, American Mathematical Monthly 71(5): 498-504.

[9] H. Tembine, E. Altman, R. El-Azouzi and Y. Hayel,Evolutionary games with random number of interacting players applied to access control, Proceedings of the 6th Intl. Symposium on Modeling and Optimization in Mobile, Ad Hoc, and Wireless Networks, WIOPT 2008.

[10] H. Tembine, E. Altman, R. ElAzouzi and Y. Hayel, A Multiple Access Game in Ad Hoc Network. Proceedings of the The First International Workshop on Game theory for Communication networks 2007.

[11] H. Tembine, E. Altman, R. ElAzouzi and Y. Hayel, Battery Statedependent Access Control in Solar-powered Broadband Wireless Networks, in Proc. of Second International EuroFGI Conference on Network Control and Optimization (NETCOOP 2008), September 2008.

[12] Yang B., Feng G., Guan X., Noncooperative Random Access Game via Pricing in Ad Hoc Networks, Proceedings of the 46th IEEE Conference on Decision and Control New Orleans, LA, USA, Dec. 12-14, 2007. 